Bull. Korean Math. Soc. 48 (2011), No. 6, pp. 1147-1155

http://dx.doi.org/10.4134/BKMS.2011.48.6.1147

\title{
FINITE NON-NILPOTENT GENERALIZATIONS OF HAMILTONIAN GROUPS
}

\author{
Zhencai Shen, Wujie Shi, And Jinshan Zhang
}

\begin{abstract}
In J. Korean Math. Soc, Zhang, Xu and other authors investigated the following problem: what is the structure of finite groups which have many normal subgroups? In this paper, we shall study this question in a more general way. For a finite group $G$, we define the subgroup $\mathcal{A}(G)$ to be intersection of the normalizers of all non-cyclic subgroups of $G$. Set $\mathcal{A}_{0}=1$. Define $\mathcal{A}_{i+1}(G) / \mathcal{A}_{i}(G)=\mathcal{A}\left(G / \mathcal{A}_{i}(G)\right)$ for $i \geq 1$. By $\mathcal{A}_{\infty}(G)$ denote the terminal term of the ascending series. It is proved that if $G=\mathcal{A}_{\infty}(G)$, then the derived subgroup $G^{\prime}$ is nilpotent. Furthermore, if all elements of prime order or order 4 of $G$ are in $\mathcal{A}(G)$, then $G^{\prime}$ is also nilpotent.
\end{abstract}

\section{Introduction}

Let $G$ be a finite group (all groups considered in this paper are finite). The notation and terminology used in this paper are standard, as in [14-16]. It is known that if $G$ normalizes each subgroup of $G$, then $G$ is a Dedekind group. We know that if $G$ normalizes all cyclic subgroups of $G$, then $G$ normalizes all subgroups of $G$. As a dual case, one can ask what can be said about the finite groups $G$ satisfying the following condition: $G$ normalizes all noncyclic subgroups of $G$ ? Note that R. Baer and H. Wielandt in 1934 and 1958, respectively, introduced the following concepts: $N(G)$ denote the intersection of the normalizers of all subgroups of $G$ and $\omega(G)$ denote the intersection of the normalizers of all subnormal subgroups of $G$. Those concepts were investigated by many authors, for example, see $[1-4,5,10,12,24$ and 26]. In fact, the generalization of the above problem had been considered by many authors, see $[6-9,17,18,20-22,25$ and 27]. In this paper, we shall study this question in a more general way. First of all, we give the following definition.

Received April 7, 2010; Revised July 9, 2011.

2010 Mathematics Subject Classification. 20D10, 20D15, 20D20, 20D30, 20F14, 20F19.

Key words and phrases. derived subgroup, meta-nilpotent group, solvable group, nilpotency class, fitting length.

The project is supported by the Natural Science Foundation of China (No. 11171364), the China Postdoctoral Science Foundation (No. 2011M500168), the NSF of Sichuan Provincial Education Department, and the NSF of Sichuan University of Science and Engineering(No. 2009xjkRL011). 
Definition 1.1. Let $\mathcal{A}(G)$ be the intersection of the normalizers of all noncyclic subgroups of $G$. That is,

$$
\mathcal{A}(G)=\bigcap_{H \in \mathcal{S}(G)} N_{G}(H),
$$

where $\mathcal{S}(G)=\{H \mid H$ is a non-cyclic subgroup of $G\}$.

Obviously, $\mathcal{A}(G)$ is a characteristic subgroup of $G$.

The case that $\mathcal{A}(G)=1$ is possible for a solvable group $G$. For instance, the symmetric group $S_{4}$ on four letters satisfies $\mathcal{A}\left(S_{4}\right)=1$.

Definition 1.2. For a group $G$, there exists a series of characteristic subgroups:

$$
1=\mathcal{A}_{0}(G) \leq \mathcal{A}_{1}(G) \leq \mathcal{A}_{2}(G) \leq \cdots \leq \mathcal{A}_{n}(G) \leq \cdots
$$

satisfying $\mathcal{A}_{i+1}(G) / \mathcal{A}_{i}(G)=\mathcal{A}\left(G / \mathcal{A}_{i}(G)\right)$ for $i=0,1,2, \ldots$ and $\mathcal{A}_{n}(G)=$ $\mathcal{A}_{n+1}(G)$ for some integer $n \geq 1$. Write $\mathcal{A}_{\infty}(G)$ for the terminal term of the ascending series.

Definition 1.3. A finite group $G$ is called a $\mathcal{A}$-group if $G=\mathcal{A}(G)$, that is, all non-cyclic subgroups of $G$ are normal.

Throughout the paper, we denote by $\mathcal{F}_{d n}$ the class of finite groups $G$ with $G^{\prime}$ nilpotent. It is well-known that $\mathcal{F}_{d n}$ is a saturated formation containing all supersolvable groups. In addition, for a $p$-group $P$ and $p$ a prime, we denote $\Omega(P)=\Omega_{1}(P)$ if $p>2$ and $\Omega(P)=\left\langle\Omega_{1}(P), \Omega_{2}(P)\right\rangle$ if $p=2$, where $\Omega_{i}(P)=\left\{x \mid x^{p^{i}}=1\right\} . \pi(G)$ denotes the set of primes dividing $|G| ; Z_{n}$ denotes the cyclic group of order $n ; Q_{8}$ denotes the quaternion group of order $8 ;[H] K$ means a split extension of a normal subgroup $H$ by a complement subgroup $K$; $G_{p}$ denotes a Sylow $p$-subgroup of $G$ for $p \in \pi(G) ; \Phi(G)$ is the Frattini subgroup of $G ; H_{G}$ is the normal core of the subgroup $H$ in $G ; l_{p}(G)$ is $p$-length; $F_{p}(G)$ is the largest normal $p$-nilpotent subgroup of $G ; O_{p^{\prime}}(G)$ is the largest normal $p^{\prime}$-subgroup of $G ; O_{p^{\prime}, p}(G)$ is the original image of $O_{p}\left(G / O_{p^{\prime}}(G)\right)$.

\section{Preliminaries}

First, we give two examples on $\mathcal{A}(G)$, which are useful in Sections 3 and 5. Example 2.1 indicates that the subgroup $\mathcal{A}(G)$ may be non-supersolvable. Example 2.2 shows that $1<\mathcal{A}(G)<G$ is possible when $G$ is a solvable group.

Example 2.1. (1) Assume that $G=A_{4}$. Then $\mathcal{A}(G)=G$;

(2) Assume that $G=\left[Q_{8}\right] Z_{3}=S L(2,3)$, where $Z_{3}$ is a cyclic subgroup of $\operatorname{Aut}\left(Q_{8}\right)$ of order 3. Then $\mathcal{A}(G)=G$ and $(\mathcal{A}(G))^{\prime}\left(=G^{\prime}\right)=Q_{8}$ is non-abelian.

Proof. (1) Since the only non-cyclic subgroups of $A_{4}$ are $A_{4}$ and $K_{4}$ (Klein 4-group), $\mathcal{A}(G)=G$.

(2) The only non-cyclic subgroups of $G$ are $G$ and $Q_{8}$. Hence $\mathcal{A}(G)=G$, and $G^{\prime}$ is non-abelian. 
Example 2.2. As Aut $\left(Q_{8}\right) \cong S_{4}$ and $D_{8} \leq S_{4}$, we have the semidirect product $G=\left[Q_{8}\right] D_{8}$. Then $G$ is a 2-group of order $2^{6}$ and $1<\mathcal{A}(G)<G$.

Proof. The derived subgroup $A$ of $D_{8}$ acts faithfully on $Q_{8}$, so $A$ is non-normal in $G$. Thus $1<\mathcal{A}(G)<G$.

The following basic properties of the subgroup $\mathcal{A}(G)$ are required in this paper.

Lemma 2.3. Let $G=A \times B$ and $(|A|,|B|)=1$. Then $\mathcal{A}(G)=\mathcal{A}(A) \times \mathcal{A}(B)$.

Proof. Let $H$ be any non-cyclic subgroup of $G$ and let $\pi$ be the set of primes dividing the order of $A$. Then $A$ is a normal Hall $\pi$-subgroup of $G$ and $B$ is a normal Hall $\pi^{\prime}$-subgroup of $G$. So $H \cap A$ is a normal Hall $\pi$-subgroup of $H$ and $H \cap B$ is a normal Hall $\pi^{\prime}$-subgroup of $H$. Therefore we have

$$
H=(H \cap A) \times(H \cap B) .
$$

Thus

$$
\begin{aligned}
N_{G}(H) & =N_{G}((H \cap A)(H \cap B)) \\
& =N_{G}((H \cap A)) \cap N_{G}((H \cap B)) \\
& \left.=\left(N_{A}(H \cap A)\right) \times B\right) \cap\left(A \times N_{B}(H \cap B)\right) \\
& =N_{A}((H \cap A)) \times N_{B}((H \cap B)) .
\end{aligned}
$$

Now the result follows.

Proposition 2.4. If $M \leq G$, then $M \cap \mathcal{A}(G) \leq \mathcal{A}(M)$.

Proof. Clearly, $M \cap \mathcal{A}(G)=M \bigcap_{H \in \mathcal{S}(G)} N_{G}(H) \leq M \bigcap_{H \in \mathcal{S}(M)} N_{G}(H)=$ $\bigcap_{H \in \mathcal{S}(M)} N_{M}(H)=\mathcal{A}(M)$.

Proposition 2.5. Let $N \leq \mathcal{A}(G)$ and $N \unlhd G$. Then $\mathcal{A}(G) / N \leq \mathcal{A}(G / N)$.

Proof. It is clear by definition.

\section{3. $\mathscr{A}_{\infty}(G)$ and $\mathcal{F}_{d n}$-groups}

Proposition 3.1. For any finite group $X$, the subgroup $\mathcal{A}(X)$ is solvable.

Proof. Write $G=\mathcal{A}(X)$. Then $G$ has the property: All non-cyclic subgroups of $G$ are normal in $G$. Consider a composition factor $K$ of $G$. Then $K$ is simple and $\mathcal{A}(K)=K$. If $H$ is any proper subgroup of $K$, we have that $H$ is cyclic. Now the theorem of Miller and Moreno [19] yields that $K$ is solvable and hence abelian. Thus all composition factors of $G$ are abelian, so $G$ is solvable.

Corollary 3.2. For any finite group $G$, the subgroup $\mathcal{A}_{\infty}(G)$ is solvable.

We can now characterize $\mathcal{F}_{d n}$-groups. 
Proposition 3.3. Let $G$ be a finite group. Then the following statements are equivalent:

(i) $G$ is an $\mathcal{F}_{d n}$-group;

(ii) $G / \mathcal{A}(G)$ is an $\mathcal{F}_{d n}$-group.

Proof. (i) $\Rightarrow$ (ii): Clear. (ii) $\Rightarrow$ (i): We use induction on the order of $G$. If $\mathcal{A}(G)=1$, then nothing needs to be shown. Suppose that $\mathcal{A}(G)>1$. So we can find a minimal normal subgroup $N$ of $G$ such that $N \leq \mathcal{A}(G)$. By Proposition $3.1, \mathcal{A}(G)$ is solvable, so $N$ is an elementary abelian $p$-group for some prime $p$.

Firstly let $N \leq \Phi(G)$. By Proposition $2.5, \mathcal{A}(G) / N \leq \mathcal{A}(G / N)$. It follows that $(G / N) / \mathcal{A}(G / N)$ is in $\mathcal{F}_{d n}$ because $G / \mathcal{A}(G) \in \mathcal{F}_{d n}$. We thus have that $G / N$ satisfies the condition of the theorem. By induction, $(G / N)^{\prime}=G^{\prime} N / N$ is nilpotent. As $N \leq \Phi(G)$, it follows by [15, III, Satz 3.5] that $G^{\prime} N$ and hence $G^{\prime}$ is nilpotent, which gives $G \in \mathcal{F}_{d n}$, as desired.

Next, let $N \nsubseteq \Phi(G)$. Then there is a maximal subgroup $M$ of $G$ such that $G=N M$ with $N \cap M=1$. By Proposition 2.4, $M \cap \mathcal{A}(G) \leq \mathcal{A}(M)$. Thus, by the hypothesis that $G / \mathcal{A}(G) \in \mathcal{F}_{d n}$, and as $G / \mathcal{A}(G) \cong M /(\mathcal{A}(G) \cap M)$, we have $M / \mathcal{A}(M) \in \mathcal{F}_{d n}$. Hence $M$ satisfies the condition. By induction, $M^{\prime}$ is nilpotent. Now $N \leq \mathcal{A}(G)$ and $\mathcal{A}(G)$ normalizes all non-cyclic subgroups of $G$. If $M$ is cyclic, then $G^{\prime} \leq N$ is a $p$-group and hence $G \in \mathcal{F}_{d n}$. Suppose that $M$ is non-cyclic, and so $N$ normalizes $M$. Thus $M$ is normal in $G$ and it follows that $G^{\prime}=N^{\prime} \times M^{\prime}$. Since $M^{\prime}$ is nilpotent, we conclude that $G^{\prime}$ is nilpotent, as desired.

Proposition 3.4. Let $G$ be a finite group and $G=\mathcal{A}_{\infty}(G)$. Then $G \in \mathcal{F}_{d n}$.

Proof. As $\mathcal{A}_{\infty}(G / \mathcal{A}(G))=\mathcal{A}_{\infty}(G) / \mathcal{A}(G)$, by induction, $G / \mathcal{A}(G) \in \mathcal{F}_{d n}$. It follows from Proposition 3.3 that $G \in \mathcal{F}_{d n}$.

Theorem 3.5. Let $G$ be a finite group and $Z(G)>1$. Then the following statements are equivalent:

(i) $G \in \mathcal{F}_{d n}$;

(ii) $G=\mathcal{A}_{\infty}(G)$.

Proof. We only need to prove (i) $\Rightarrow$ (ii). Since $Z(G)>1, \mathcal{A}(G)>1$. It is clear that $G / \mathcal{A}(G) \in \mathcal{F}_{d n}$. Thus, by induction, we have $G / \mathcal{A}(G)=\mathcal{A}_{\infty}(G / \mathcal{A}(G))$. Moreover, $\mathcal{A}_{\infty}(G / \mathcal{A}(G))=\mathcal{A}_{\infty}(G) / \mathcal{A}(G)$ gives that $G=\mathcal{A}_{\infty}(G)$.

\section{4. $\mathscr{A}$-groups}

The following facts are clear from Definition 1.3:

Proposition 4.1. (i) The subgroups of a $\mathcal{A}$-group are $\mathcal{A}$-groups;

(ii) The quotient groups of a $\mathcal{A}$-group are $\mathcal{A}$-groups.

By Proposition 2.3, we have: 
Theorem 4.2. If $G$ is a finite nilpotent group, then $G$ is a $\mathcal{A}$-group if and only if all Sylow subgroups of $G$ are $\mathcal{A}$-groups.

Remark. Finite nilpotent $\mathcal{A}$-groups were considered by Passman, Bozikov, Janko, Song and $\mathrm{Qu}$, see [8, 22 and 25]. In addition, finite non-nilpotent $\mathcal{A}$-groups were considered by Zhang, Guo, $\mathrm{Qu}$ and $\mathrm{Xu}$, see [27].

For convenience of the readers, we give the following:

Theorem 4.3. $G$ is a finite non-nilpotent $\mathcal{A}$-group if and only if $G$ is one of the following groups:

(i) $\left[Z_{p}\right] Z_{n}$, where $p$ is a prime and $Z_{n}$ is not normal in $G$;

(ii) $\left[Z_{p}^{2}\right] Z_{n}$, where $p$ is a prime, $(p, n)=1$ and $Z_{n}$ acts irreducibly on $Z_{p}^{2}$;

(iii) $\left(\left[Q_{8}\right] Z_{3^{m}}\right) \times Z_{n}$, where $(6, n)=1$ and $Q_{8}$ is not normal in $\left[Q_{8}\right] Z_{3^{m}}$.

\section{Applications}

Gaschütz and Itô proved that if all minimal subgroups of a group $G$ are normal (in which case $G$ is called a PN-group), then $G$ is solvable and the Fitting length of $G$ is at most 3 ([15, p. 436, Theorem 5.7 or 11, Theorem 1]). In this section, the following dual problem is considered: study the finite group $G$ all of whose minimal subgroups normalize every non-cyclic subgroup of $G$.

Theorem 5.1. Let $G$ be a p-solvable group. Suppose that all elements of $G$ of order $p$ are in $\mathcal{A}(G)$. If $p=2$, suppose in addition that all elements of $G$ of order 4 are in $\mathcal{A}(G)$. Then $l_{p}(G) \leq 1$.

Proof. We use induction on $|G|$. Clearly, $G / O_{p^{\prime}}(G)$ satisfies the hypothesis and $l_{p}\left(G / O_{p^{\prime}}(G)\right)=l_{p}(G)$. So we may assume that $O_{p^{\prime}}(G)=1$.

Let $P$ be a Sylow $p$-subgroup of $\mathcal{A}(G)$. By Proposition 3.3, $\mathcal{A}(G)^{\prime}$ is nilpotent. Thus $O_{p^{\prime}}(G)=1$ implies $\mathcal{A}(G)^{\prime}$ is a $p$-group, and hence $P$ is normal in $G$. Also, $F_{p}(G)=O_{p^{\prime}, p}(G)=O_{p}(G)$. As $G$ is $p$-solvable, by [23, p. 269, Theorem 9.3.1], we know

$$
C_{G}\left(O_{p}(G)\right) \leq O_{p}(G) \text {. }
$$

We now claim that $G$ is $q$-nilpotent for any prime $q \neq p$. Otherwise, there exists a prime $q$ such that $G$ is non- $q$-nilpotent. Then there exists a subgroup $K$ with the following properties: $K$ is non- $q$-nilpotent but all proper subgroups of $K$ are $q$-nilpotent. By a theorem of Itô [23, p. 296, Theorem 10.3.3], $K$ has a normal Sylow $q$-subgroup $Q$ and $\exp (Q)=q$ or 4 . By above, $\Omega\left(G_{p}\right) \leq P \leq O_{p}(G)$, so $\Omega\left(G_{p}\right)=\Omega\left(O_{p}(G)\right)$. Since $K$ is non-cyclic, by hypothesis, $\Omega\left(O_{p}(G)\right)$ normalizes $K$. On the other hand, since $Q \unlhd K$, it follows that $\Omega\left(O_{p}(G)\right)$ normalizes $Q$ and $\left[Q, \Omega\left(O_{p}(G)\right)\right]=1$. By $[15$, p. $437,5.12]$, we get $\left[Q, O_{p}(G)\right]=1$. Thus $Q \leq C_{G}\left(O_{p}(G)\right)$. As $C_{G}\left(O_{p}(G)\right) \leq O_{p}(G)$ and $Q$ is a $p^{\prime}$-group, $Q$ must be 1 , a contradiction.

Now let $G_{q^{\prime}}$ denote the normal $q$-complement of $G$ for every prime $q \neq p$. Then $G_{p} \leq G_{q^{\prime}}$ and $G_{p}$ is the intersection of all $G_{q^{\prime}}$, hence $G_{p} \unlhd G$, of course, $l_{p}(G)=1$. The proof is now complete. 
Theorem 5.2. Let $G$ be a finite group. If all elements of $G$ of prime order are in $\mathcal{A}(G)$, then $G$ is solvable.

Proof. Assume that the theorem is false and let $G$ be a counterexample of minimal order. If $M$ is a proper subgroup of $G$, by Proposition 2.4 we have $M \cap \mathcal{A}(G) \leq \mathcal{A}(M)$. Thus all subgroups of $M$ of prime order are in $\mathcal{A}(M)$. So $M$ satisfies the condition. By the choice of $G, M$ is solvable. Consequently, $G$ is a non-solvable group in which all proper subgroups are solvable, by [13, Theorem 4.1], $G / \Phi(G)$ is a minimal simple group. As $\mathcal{A}(G)$ is normal in $G$ and solvable, it follows that $\mathcal{A}(G) \leq \Phi(G)$.

Let $p$ be an odd prime dividing the order of $G$. We claim:

(1) $\Omega_{1}\left(G_{p}\right) \unlhd G$.

It is well known that $\Phi(G)$ is nilpotent, so all Sylow subgroups of $\Phi(G)$ are normal in $G$. Let $P$ be the Sylow $p$-subgroup of $\Phi(G)$. By the hypothesis, all subgroups of $G$ of order $p$ are in $\mathcal{A}(G)$ and hence in $P$, so $\Omega_{1}\left(G_{p}\right)=\Omega_{1}(P)$. Thus $\Omega_{1}\left(G_{p}\right)$ char $P \unlhd G$, (1) follows.

(2) $C_{G}\left(\Omega_{1}\left(G_{p}\right)\right) \leq \Phi(G)$.

By (1), $\Omega_{1}\left(G_{p}\right)$ is normal in $G$, so it follows that $C_{G}\left(\Omega_{1}\left(G_{p}\right)\right)$ is normal in $G$. Thus $G / \Phi(G)$ contains a normal subgroup $C_{G}\left(\Omega_{1}\left(G_{p}\right)\right) \Phi(G) / \Phi(G)$. As $G / \Phi(G)$ has no non-trivial normal subgroups, we have $C_{G}\left(\Omega_{1}\left(G_{p}\right)\right) \Phi(G)=$ $\Phi(G)$ or $C_{G}\left(\Omega_{1}\left(G_{p}\right)\right) \Phi(G)=G$. Suppose that the second case happens. Then we have $C_{G}\left(\Omega_{1}\left(G_{p}\right)\right)=G$, i.e., $\Omega_{1}\left(G_{p}\right) \leq Z(G)$. Thus all elements of $G$ of order $p$ are in $Z(G)$. Noting that $p$ is an odd prime, we can apply the Itô lemma $[15$, p. 435 , Theorem 5.5] to see that $G$ is $p$-nilpotent. Because the quotient groups of a $p$-nilpotent group are also $p$-nilpotent, we see that $G / \Phi(G)$ would be $p$-nilpotent. But $G / \Phi(G)$ has no non-trivial normal subgroup, which implies that $G / \Phi(G)$ is a $p^{\prime}$-group. However, by [15, III, Theorem 3.8], $p|| G / \Phi(G) \mid$ holds whenever $p|| \Phi(G) \mid$. This is a contradiction. We thus conclude that only the first case is true, which implies (2).

Fix an odd prime $p$ as above. Consider the subgroup

$$
N=N_{G}\left(G_{p}\right) \text {. }
$$

By the Schur-Zassenhaus theorem [23, p. 253, Theorem 9.1.2], $N$ possesses a Hall $p^{\prime}$-subgroup $H$ such that $N=\left[G_{p}\right] H$. By the condition, $\Omega_{1}\left(G_{p}\right) \leq \mathcal{A}(G)$.

(3) $H^{\prime} \leq \Phi(G)$ and $N^{\prime}=G_{p}^{\prime} \times H^{\prime}$.

Case 1. If $H$ is cyclic, then $N^{\prime} \leq G_{p}^{\prime}$. Moreover, as $G_{p}$ is a subgroup of $N$, it follows that $G_{p}^{\prime} \leq N^{\prime}$. Thus $G_{p}^{\prime}=N^{\prime}$.

Case 2. If $H$ is non-cyclic, then by the hypotheses $\Omega_{1}\left(G_{p}\right)$ normalizes $H$. On the other hand, by $(1)$, we have $\Omega_{1}\left(G_{p}\right) \unlhd N$. Thus $\left[\Omega_{1}\left(G_{p}\right), H\right] \leq \Omega_{1}\left(G_{p}\right) \cap H=$ 1 and $H$ acts trivially on $\Omega_{1}\left(G_{p}\right)$ by conjugation. Hence $H \leq C_{G}\left(\Omega_{1}\left(G_{p}\right)\right) \leq$ $\Phi(G)$ and of course, $H^{\prime} \leq \Phi(G)$. Moreover, by [15, p. 437, 5.12], $H$ acts trivially on $G_{p}$. That is, $G_{p} H=G_{p} \times H$, so $N=G_{p} \times H$ and

$$
N^{\prime}=G_{p}^{\prime} \times H^{\prime}
$$

(4) $G_{p}^{\prime} \leq \Phi(G)$, in particular, $N^{\prime} \leq \Phi(G)$. 
As $G$ is non-solvable, there exists another odd prime $q$ dividing the order $G$ such that $q \neq p$. Let $G_{q}$ be a Sylow $q$-subgroup of $G$. By (1), we have $\Omega_{1}\left(G_{q}\right) \unlhd G$. Also, by the hypothesis, $\Omega_{1}\left(G_{q}\right) \leq \mathcal{A}(G)$. If $G_{p}$ is cyclic, then $G_{p}^{\prime}=1 \leq \Phi(G)$. If $G_{p}$ is non-cyclic, then $\Omega_{1}\left(G_{q}\right)$ normalizes $G_{p}$. Thus $\Omega_{1}\left(G_{q}\right) G_{p}=\Omega_{1}\left(G_{q}\right) \times G_{p}$, and hence $C_{G}\left(\Omega_{1}\left(G_{q}\right)\right) \geq G_{p}$. Applying $(2)$, we see that $G_{p} \leq \Phi(G)$. Therefore, $G_{p}^{\prime} \leq \Phi(G)$, as desired.

(5) The final contradiction.

Let $\bar{G}=G / \Phi(G)$. Then $\bar{G}_{p}=G_{p} \Phi(G) / \Phi(G)$ is a Sylow $p$-subgroup of $G / \Phi(G)$. Write $N_{\bar{G}}\left(\bar{G}_{p}\right)=M / \Phi(G)$. Then $G_{p} \Phi(G) \unlhd M$ and $G_{p}$ is a Sylow $p$ subgroup of $G_{p} \Phi(G)$. By the Frattini argument $M=N_{M}\left(G_{p}\right) \Phi(G)$. Therefore $N_{\bar{G}}\left(\bar{G}_{p}\right)=N_{G}\left(G_{p}\right) \Phi(G) / \Phi(G)$. Now, $N_{\bar{G}}\left(\bar{G}_{p}\right) \cong N_{G}\left(G_{p}\right) /\left(N_{G}\left(G_{p}\right) \cap \Phi(G)\right)$, and, by (4), $N_{G}\left(G_{p}\right)^{\prime}=N^{\prime} \leq \Phi(G)$, so $N_{G}\left(G_{p}\right) /\left(N_{G}\left(G_{p}\right) \cap \Phi(G)\right)$ is abelian. Consequently, $N_{\bar{G}}\left(\bar{G}_{p}\right)$ is abelian. By a theorem of Burnside [15, IV, Theorem 2.6], $\bar{G}$ is $p$-nilpotent. This is impossible because $\bar{G}$ is a minimal simple group. The proof now is complete.

Theorem 5.3. Let $G$ be a finite group. If all elements of $G$ of prime order or order 4 are in $\mathcal{A}(G)$, then $G^{\prime}$ is nilpotent.

Proof. Let $p$ be any prime dividing $|G|$ and let $P$ be a Sylow $p$-subgroup of $G$. As $G$ is solvable, it is $p$-solvable. According to Theorem 5.1, we have $F_{p}(G)=O_{p^{\prime}, p}(G)=O_{p^{\prime}}(G) P$, the maximal normal $p$-nilpotent subgroup of $G$. Next, by the Frattini argument $G=N_{G}(P) O_{p^{\prime}}(G)$. On the other hand, by the Schur-Zassensaus theorem [23, p. 253, Theorem 9.1.2], $N_{G}(P)=[P] M$, where $M$ is a Hall $p^{\prime}$-subgroup of $N_{G}(P)$ and hence $G=F_{p}(G) M$.

If $M$ is cyclic, then $G^{\prime} \leq F_{p}(G) M^{\prime}=F_{p}(G)$ and $G^{\prime}$ is $p$-nilpotent.

If $M$ is non-cyclic, by the hypothesis, $\Omega_{1}(P)$ and $\Omega_{2}(P)$ normalize $M$. Hence $M$ centralizes $\Omega_{1}(P)$ and $\Omega_{2}(P)$, and thus centralizes $P$. Since $C_{G}(P) \leq F_{p}(G)$ by [23, p. 269, Theorem 9.3.1],

$$
M \leq F_{p}(G) .
$$

Now $G=F_{p}(G) M$, so it follows that $G=F_{p}(G)$. Of course, $G^{\prime}$ is $p$-nilpotent. Hence $G^{\prime}$ is nilpotent.

Theorem 5.4. Let $G$ be a finite group. If all elements of $G$ of prime order or order 4 are in $\mathcal{A}(G)$, then

(i) $G$ is solvable;

(ii) $l_{p}(G) \leq 1$ for every prime $p$, and

(iii) the Fitting length of $G$ is bounded by 2.

Proof. This follows from Theorems 5.1, 5.2 and 5.3.

Let us compare Theorem 5.4 with the following well-known result: If all the cyclic subgroups of a group $G$ of prime order or order 4 are normal, then $G$ is supersolvable [11]. The previous Example 2.1 shows that the supersolvable conclusion cannot be expected under the condition of Theorem 5.4. 
Acknowledgments. The authors are grateful to the referee and the editor who provided their valuable suggestions and help. In particular, the authors are grateful to the referee who provided a detailed report. The authors are grateful to Profs. M. F. Newman, Z. Bozikov, Z. Janko and M. Xu who provided their help.

\section{References}

[1] R. Baer, Der Kern eine charakteristische Untergruppe, Compositio Math. 1 (1934), $254-283$.

[2] — Norm and hypernorm, Publ. Math. Debrecen 4 (1956), 347-356.

[3] $—$ Classes of finite groups and their properties, Illinois J. Math. 1 (1957), 115-187.

[4] _ Zentrum und Kern von Gruppen mit Elementen unendlicher Ordnung, Compositio Math. 2 (1935), 247-249.

[5] J. C. Beidleman, H. Heineken, and M. Newell, Centre and norm, Bull. Austral. Math. Soc. 69 (2004), no. 3, 457-464.

[6] T. R. Berger, L. G. Kovács, and M. F. Newman, Groups of prime power order with cyclic Frattini subgroup, Nederl. Akad.Wetensch. Indag. Math. 42 (1980), no. 1, 13-18.

[7] Y. Berkovich and Z. Janko, Structure of finite p-groups with given subgroups, Ischia group theory 2004, 13-93, Contemp. Math., 402, Amer. Math. Soc., Providence, RI, 2006.

[8] Z. Bozikov and Z. Janko, A complete classification of finite p-groups all of whose noncyclic subgroups are normal, Glas. Mat. Ser. III 44(64) (2009), no. 1, 177-185.

[9] R. Brandl, Groups with few non-normal subgroups, Comm. Algebra 23 (1995), no. 6, 2091-2098.

[10] R. A. Bryce and J. Cossey, The Wielandt subgroup of a finite soluble group, J. London Math. Soc. (2) 40 (1989), no. 2, 244-256.

[11] J. Buckley, Finite groups whose minimal subgroups are normal, Math. Z. 116 (1970), no. $1,15-17$.

[12] A. R. Camina, The Wielandt length of finite groups, J. Algebra 15 (1970), 142-148.

[13] Z. Chen, Inner $\Sigma$-groups. II, Acta Math. Sinica 24 (1981), no. 3, 331-336.

[14] D. Gorenstein, Finite Groups, Chelsea, New York, 1980.

[15] B. Huppert, Endliche Gruppen I, Springer-Verlag, Berlin, Heidelberg, New York, 1967.

[16] H. Kurzweil and B. Stellmacher, The Theory of Finite Groups: An Introduction, Springer-Verlag New York, Inc., 2004.

[17] S. Li and Z. Shen, On the intersection of normalizers of direved subgroups of all subgroups of a finite group, J. Algebra 323 (2010), 1349-1357.

[18] T. D. Lukashova, On the noncyclic norm of infinite locally finite groups, Ukrain. Mat. Zh. 54 (2002), no. 3, 342-348; translation in Ukrainian Math. J. 54 (2002), no. 3, 421428.

[19] G. A. Miller and H. C. Moreno, Non-abelian groups in which every subgroup is abelian, Trans. Amer. Math. Soc. 4 (1903), no. 4, 398-404.

[20] H. Mousavi, On Finite groups with few non-normal subgroups, Comm. Algebra 27 (1999), no. 7, 3143-3151.

[21] M. Newman and J. Wiegold, Groups with many nilpotent subgroups, Arch. Math. 15 (1964), 241-250.

[22] D. S. Passman, Nonnormal subgroups of p-groups, J. Algebra 15 (1970), 352-370.

[23] D. J. S. Robinson, A Course in the Theory of Groups, Springer-Verlag, New York, 1982.

[24] E. Schenkman, On the norm of a group, Illinois J. Math. 4 (1960), 150-152.

[25] Q. Song and H. Qu, Finite 2-groups whose subgroups are cyclic or normal, Math. Pract. Theory 38 (2008), no. 10, 191-197. 
FINITE NON-NILPOTENT GENERALIZATIONS OF HAMILTONIAN GROUPS 1155

[26] H. Wielandt, Über der Normalisator der Subnormalen Untergruppen, Math. Z. 69 (1958), 463-465.

[27] Q. Zhang, X. Guo, H. Qu, and M. Xu, Finite Groups which have many normal subgroups, J. Korean Math. Soc. 46 (2009), no. 6, 1165-1178.

ZHENCAI SHEN

LMaM and School of Mathematical Sciences

Peking University

Beijing, 100871, P. R. China

E-mail address: zhencai688@sina.com

WujIE SHI

School of Mathematics and Statics

Chongqing University of Arts and Sciences

Chongqing, 402160, P. R. China

E-mail address: wjshi@suda.edu.cn

JINSHAN ZHANG

SCHOOL OF SCIENCE

Sichuan University of Science and Engineering

Zigong, 643000, P. R. ChinA

E-mail address: zjscdut@163.com 